\title{
Correspondence
}

The Editor,

journal of Glaciology

SIR,

\section{Horizontal shear rate of ice initially exhibiting vertical compression fabrics}

Typically, the pattern of crystal-orientation fabric found in the ice cores retrieved from polar ice sheets varies from random in the surface layers to the development with depth of systematic patterns resulting from increasing stress and strain. In accumulation areas vertical compression tends to be the dominant form of deformation near the surface. Below the surface layers, vertical compression can yield a small-circle girdle pattern, a two-maxima pattern, some intermediate pattern depending on the relative magnitude of the longitudinal and transverse strains (Budd and Jacka, 1989), or a girdle-like rotation fabric at stresses and temperatures lower than those required for formation of a true girdle (Alley, 1992). By about one-third of the depth or more (depending on the site), horizontal shear begins to dominate, ultimately resulting in the development of a fabric pattern characterized by a high concentration of vertical $c$ axes.

For accurate modelling of ice-sheet flow, account needs to be taken of these anisotropic crystal-fabric patterns since they have a significant effect on the flow rate. For example, we know that uniaxial compression of ice parallel to the symmetry axis of a small-circle girdle fabric will result in a flow rate up to three times greater than the flow rate of isotropic ice. Simple shear deformation of ice exhibiting a single maximum orientation fabric (with $c$ axes perpendicular to the shear direction, i.e. basal (glide) planes parallel to the shear direction) will result in flow rates up to ten times greater than for isotropic ice (Budd and Jacka, 1989; LiJun and others, 1996).

These combinations of deformation configuration and crystal orientation, which result in flow rates greater than the isotropic flow rate, are termed "easy glide". Other combinations (e.g. compression parallel to the central axis of a single maximum fabric) can result in "hard glide" relative to the isotopic ice-flow rates.

Of interest here is the combination involving horizontal shear of ice initially exhibiting crystal fabrics that have resulted from vertical compression. It is important that this combination be understood, because it is the situation in ice sheets in the region where shear begins to dominate over compression. Because the median $c$-axis angle to the vertical for the compression fabrics is typically $<40^{\circ}$, this combination is expected to result in easy glide. A brief discussion at the European Ice Sheet Modelling Initiative (EISMINT) Workshop on Ice Rheology at Grindelwald, Switzerland, in 1997 agreed that a laboratory check of this was warranted. Until now, to our knowledge, no experimental confirmation of this has been provided and no flow rates for this configuration have been measured in the laboratory. We have now carried out a series of ice-deformation tests at $-2^{\circ} \mathrm{C}$ aimed at measuring these flow rates.

Our creep deformation apparatus (Li Jun and others, 1996) allows us to apply compression or tension, simple shear and simultaneous combinations of compression (or tension) and shear. Importantly for this study, it also allows us to test in one stress configuration, and to follow this with a test on the same ice sample in another configuration. We can test samples of different shapes and sizes. Since sample shape is distorted by deformation to high strains (e.g. an initially cylindrical sample in compression will "barrel"), it is important also that we can remove a tested sample from the apparatus, turn it back to its original shape, then reload and retest. For a cylindrical sample in compression, the sample can also be cut in half for thin-section sampling perpendicular to the compression axis. After thin-section sampling the sample can be cemented together again by placing a drop of water between the two halves, then retested. For a rectangular oblong sample tested in simple shear, thin-section sampling through the centre of the sample is usually carried out parallel to the shear direction. Even if the two halves could be cemented back together, the accuracy of shear-deformation results parallel to the cut could not be assured.

We began work for this study by carrying out four tests in uniaxial compression, on laboratory-prepared smallgrained isotropic ice samples (Jacka and Lile, 1984). A summary of all tests carried out is provided in Table l. Two identical tests were carried out in unconfined vertical compression under an octahedral (root mean square of principal deviator tensors) stress of $0.2 \mathrm{MPa}$, on cylindrical samples (30 mm long in the vertical; $25.4 \mathrm{~mm}$ diameter) to $15 \%$ octahedral strain. One of these samples was then removed from the apparatus for thin-section analysis, while the other was left in the apparatus for testing in simple shear. The crystal-orientation fabric resulting from the compression was a small-circle girdle with symmetry axis parallel to the compression direction and a median $c$-axis angle to the vertical of $38^{\circ}$. Another two identical compression tests were performed on rectangular oblong samples $(140 \mathrm{~mm} \times$ $15 \mathrm{~mm} \times 25 \mathrm{~mm}$ ) at $0.3 \mathrm{MPa}$ octahedral, to $15 \%$ octahedral strain. Again, at the conclusion of these tests, one sample was removed for thin-section analysis, while the other was left in the apparatus for testing in simple shear. Vertical compression perpendicular to the long dimension of a rectangular oblong sample generates deformation outwards, i.e. in the two horizontal directions. For a sample significantly longer than it is wide, the horizontal deformation is primarily in the short dimension; deformation in the long dimension is relatively small. That is, the deformation is largely confined to two dimensions ( $\mathrm{Li}$ Jun and others, 1996). The resulting crystal fabric indicated a two-maxima pattern with a median $c$-axis angle to the vertical of $41^{\circ}$. We assume that the crystal-fabric patterns from the measured samples are the same as those of their identical tests described above.

The two samples remaining in the deformation apparatus were next tested in simple shear in the horizontal plane at the same temperature $\left(-2^{\circ} \mathrm{C}\right)$ and at the same octahedral stresses (0.2 MPa for the small-circle fabric sample; $0.3 \mathrm{MPa}$ for the two-maxima sample: tests B in Figs 1 and 2, respectively). For each of these stresses for comparison purposes, we also shear-tested a new laboratory-prepared isotropic sample of the same shape and size (tests A in Figs 1 and 2). For the rectangular oblong samples, yet another test was in- 


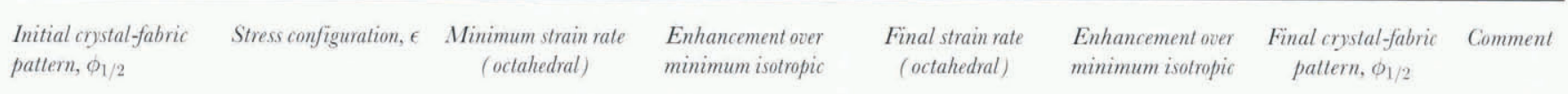

Temperature $-2^{\circ} \mathrm{C}$; stress $0.2 \mathrm{MPa}$ octahedral; cylindrical samples; Fig. I

\begin{tabular}{|c|c|c|c|c|c|c|c|}
\hline Isotropic, $57^{\circ}$ & Compression, $15 \%$ & & & & & Small circle, $38^{\circ}$ & \\
\hline Isotropic, $57^{\circ}$ & Compression, $15 \%$ & & & & & Small circle ${ }^{*}, 38$ & Retested \\
\hline Small circle $e^{*}, 38$ & Shear, $30 \%$ & $2.0 \times 10^{-7} \mathrm{~s}^{-1}$ & 2.5 & $5.8 \times 10^{-7} \mathrm{~s}^{-1}$ & 7.3 & Single pole, $32^{\circ}$ & Test B \\
\hline Isotropic, $57^{\circ}$ & Shear, $30 \%$ & $8.0 \times 10^{8} \mathrm{~s}^{1}$ & 1.0 & $5.0 \times 10^{-7} \mathrm{~s}^{-1}$ & 6.3 & Single pole, 35 & Test A \\
\hline \multicolumn{8}{|c|}{ Temperature $-2 \mathrm{C}$; stress $0.3 \mathrm{MPa}$ octahedral; rectangular oblong samples; Fig. 2} \\
\hline Isotropic, $61^{\circ}$ & Compression, $15 \%$ & & & & & Two-maxima & \\
\hline Isotropic, $61^{\circ}$ & Compression, $15 \%$ & & & & & Two-maxima* ${ }^{*} 41$ & Retested \\
\hline Two-maxima ${ }^{*}, 41^{\circ}$ & Shear, $30 \%$ & $3.55 \times 10^{-7} \mathrm{~s}^{-1}$ & 2.3 & $1.62 \times 10^{6} \mathrm{~s}^{-1}$ & 10.5 & Single pole, $31^{\circ}$ & Test B \\
\hline Isotropic, $61^{\circ}$ & Shear, $30 \%$ & $1.54 \times 10^{-7} \mathrm{~s}^{1}$ & 1.0 & $1.62 \times 10^{-6} \mathrm{~s}^{-1}$ & 10.5 & Single pole, $20^{\circ}$ & Test A \\
\hline Isotropic, $61^{\circ}$ & Shear, $30 \%$ & & & & & Single pole ${ }^{*}, 20^{\circ}$ & Retested \\
\hline Single pole ${ }^{*}, 20^{\circ}$ & Shear, $30 \%$ & $1.00 \times 10^{6} \mathrm{~s}^{-1}$ & 6.5 & $1.75 \times 10^{-6} \mathrm{~s}^{-1}$ & 11.4 & Single pole, $17^{\circ}$ & Test C \\
\hline
\end{tabular}

$\phi_{1 / 2}$ is the median crystal $c$-axis angle to the vertical. $\epsilon$ is total strain attained by the test. Asterisks indicate that the final crystal-fabric pattern is assumed on the basis of the identical test listed immediately above. Each of the samples marked with an asterisk was retested as shown in the row immediately below.

cluded. A sample (initially isotropic and of the same shape and size as the others) which had previously been deformed in shear at $0.3 \mathrm{MPa}$ to $30 \%$ octahedral was removed from the apparatus, recut to its initial shape, then returned to the apparatus for retesting (test $\mathrm{C}$ in Fig. 2). The initial crystal structure for this test is assumed the same as the final crystal structure of test A.

Results of the shear tests are summarized (plots of octahedral strain rate as a function of strain) in Figure 1 (the cylindrical samples at $0.2 \mathrm{MPa}$ ) and Figure 2 (the rectangular oblong samples at $0.3 \mathrm{MPa}$ ), along with crystal-orientation fabric diagrams plotted before (i.e. from the identical samples as described above) and after each test. The strain rate for the initially isotropic samples (tests $\mathrm{A}$ in both Figures 1 and 2) decreased to a minimum, then increased with strain to a steady-state tertiary value a factor of $\sim 10$ greater than the minimum. Previous studies in compression (Jacka and Maccagnan, 1984) and shear (Gao, 1992) have shown for an initially isotropic sample that the ice is still isotropic at minimum strain rate and that crystal anisotropy then develops during the accelerating stage of the creep curve (i.e. between the isotropic minimum and the tertiary strain rates). This minimum isotropic strain rate is the value against which other strain rates may be rated as easy glide or hard glide. The crystal fabrics at the conclusion of these tests indicated development of a single maximum pattern, compatible with easy glide in simple shear. Note, however, that these single maximum patterns are slightly elongated perpendicular to the shear direction. This has resulted from the sample geometry, which allows some deformation perpendicular to the shear direction. We suggest that in a much larger (wider) sample, this effect would be evident only near the edges of the sample. In an infinitely large sample (e.g. a polar ice mass with no transverse strain) this effect is eliminated. For test $\mathrm{B}$ of Figure 2, notice also from the $c$-axis pattern some remnant evidence of the initial fabric.

Test C of Figure 2 shows strain rates decreasing (recovery of the anelastic component of the creep) to a minimum value a factor of 6.5 greater than the minimum isotropic strain rate. This is a factor of $<2$ less than the steady-state strain rate which is very similar to that ultimately attained by test $\mathrm{A}$. This result is expected since the initial crystal fabric for test $\mathrm{C}$ was close to (but not quite)

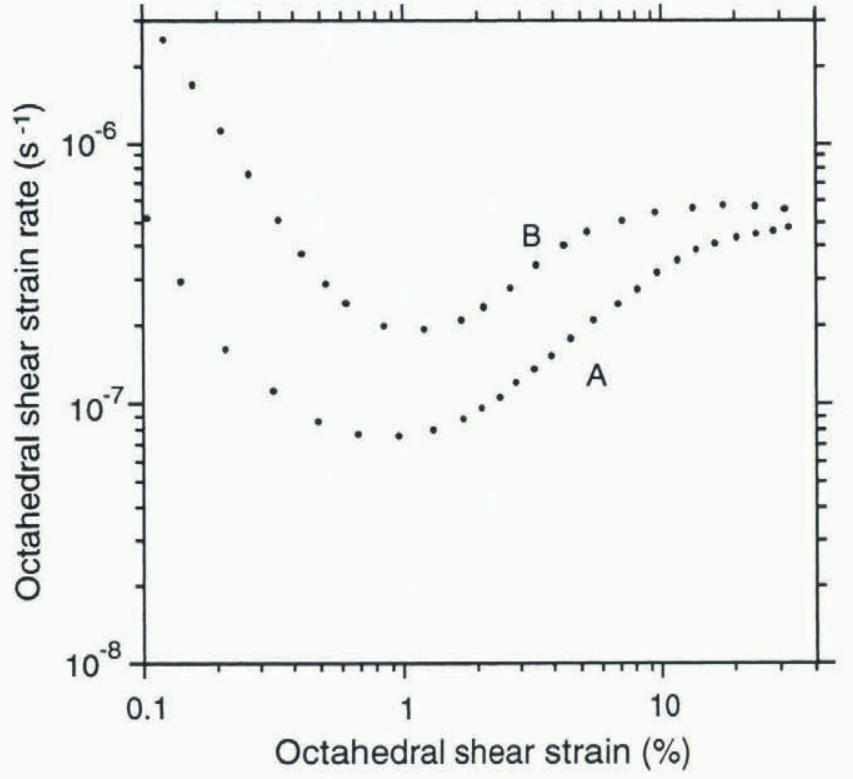

A
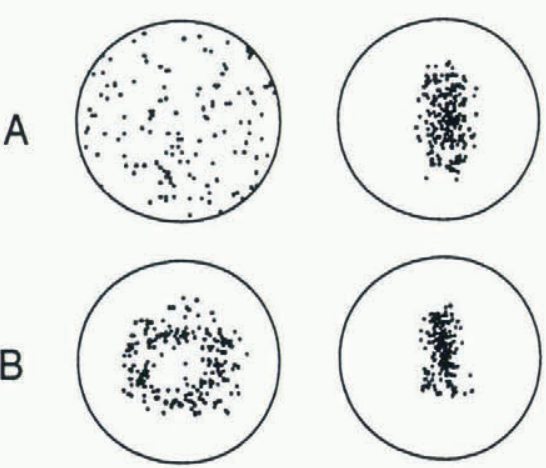

Fig. 1. Creep curves for deformation tests on cylindrical samples in simple shear at $-2^{\circ} \mathrm{C}$ and $0.2 \mathrm{MPa}$. Samples were initially isotropic (test $A$ ), and exhibiting a small-circle crystal-fabric pattern (test B). Also shown are the Schmidt equalarea $c$-axisfabric diagrams at the start (left) and conclusion (right) of the tests. For the fabric diagrams, the shear direction is to the right.

the steady-state fabric for this stress configuration. If the initial fabric had been developed a little further i.e. a few more per cent strain in the initial test), we suggest that there 


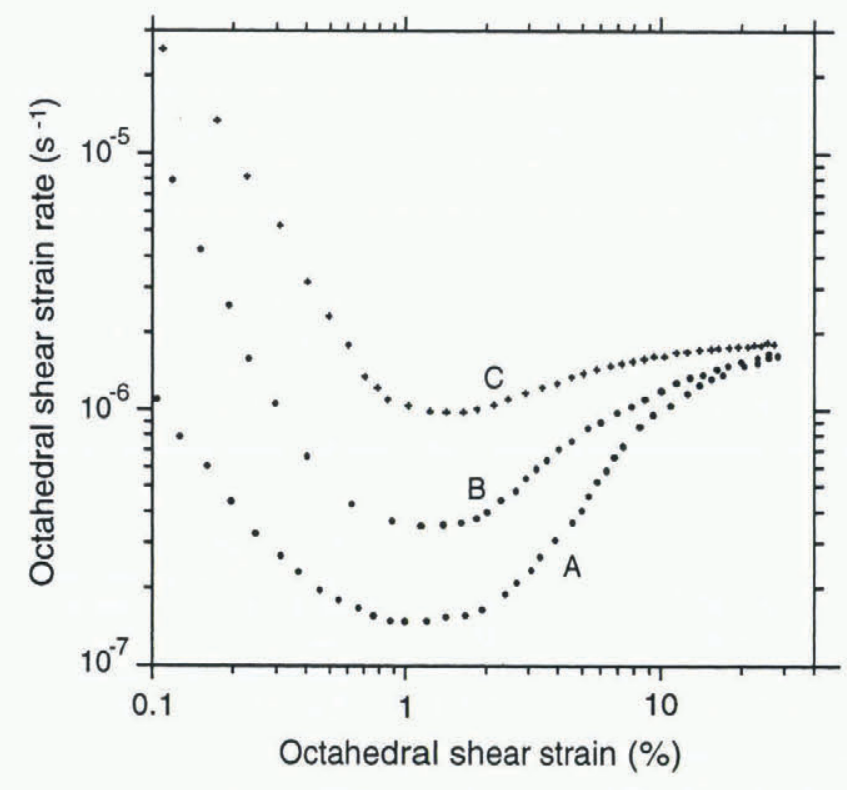

A
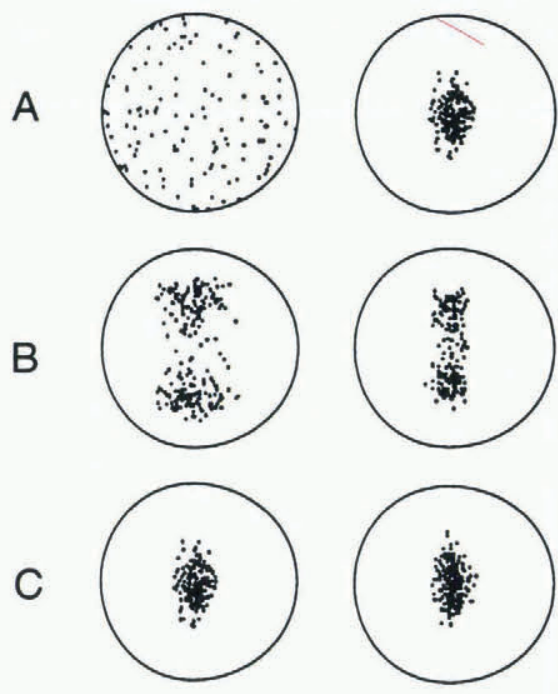

Fig. 2. Creep curves for deformation tests on rectangular oblong samples in simple shear at $-2^{\circ} \mathrm{C}$ and $0.3 \mathrm{MPa}$. Samples were initially isotropic (test $A$ ), exhibiting a two-maxima crystalfabric pattern (test $B$ ), and exhibiting a single-pole crystalfabric pattern (test C). Also shown are the Schmidt equalarea $c$-axis fabric diagrams at the start (left) and conclusion (right) of the tests. For the fabric diagrams, the shear direction is to the right, and for the initial sample of test $B$, the sample length was aligned left to right during the compression test.

would have been no minimum strain rate: the creep curve would have approached directly to the steady-state strainrate value. The crystal-fabric pattern at the conclusion of this test was similar to but slightly stronger (the median $c$-axis angle to the vertical changes from $20^{\circ}$ to $17^{\circ}$ ) than the initial pattern.

Tests B (horizontal shear on the ice with vertical compression fabrics) show strain rates decreasing to a minimum value, then increasing with strain, again to a steady-state value similar to that in the other tests. The minimum strain rate is (for both cases) a factor of $\sim 2.5$ greater than the isotropic minimum strain rate; i.e shear of ice exhibiting a small-circle girdle or a two-maxima fabric pattern, in the direction perpendicular to the symmetry axis of the fabric, generates easy glide with a strain rate a factor of $\sim 2.5$ greater than the minimum isotropic strain rate. With increasing strain, the strain rate increases to a tertiary rate similar to those for the other shear tests. The crystal fabric at the conclusion of this test, again, is similar to the fabric at the conclusion of the other two tests.

\section{CONCLUSION}

Vertical compression fabrics after large strain tend to have a degree of concentration of $c$ axes towards the vertical characterized by (1) in unconfined compression, at higher stresses and temperatures leading to recrystallization, a smallcircle girdle pattern with median angle to the vertical of $\sim 25^{\circ}$ to $40^{\circ}$; (2) in compression confined in the line of flow, again at higher temperatures and stresses leading to recrystallization, a two-maxima pattern with similar median angle; or (3) a girdle-like rotation fabric generated at lower stresses and temperatures than required for formation of a true girdle.

The horizontal shear rates of samples with vertical compression fabrics exhibit flow enhancements of $\sim 2.5$ relative to the minimum strain rates for isotropic ice. With increasing shear strain the enhancement increases to $\sim 10$ with the development of a strong single maximum fabric. These fabric and strain-rate developments are similar to those occurring in ice sheets from vertical compression near the surface to high shear with increasing depth

Antartic CRC and

LIJUN

Australian Antarctic Division,

Box 252-80,

Hobart,

Tasmania 7001,

Australia

\section{September 1998}

\section{REFERENCES}

Alley, R.B. 1992. Flow-law hypotheses for ice-sheet modeling. J. Glaciol., 38 (129), 245-256.

Budd, W. F. and T. H. Jacka. 1989. A review of ice rheology for ice sheet modelling. Cold Reg. Sci. Technol., 16 (2), 107-144.

Gao, X. Q. 1992. Laboratory studies of the development of anisotropic crystal structure and the flow properties of ice. (Ph.D. thesis, University of Melbourne.)

Jacka, T. H. and R. C. Lile. 1984. Sample preparation techniques and compression apparatus for ice flow studies. Cold Reg. Sci. Technol., 8 (3), 235-240.

Jacka, T. H. and M. Maccagnan. 1984. Ice crystallographic and strain rate changes with strain in compression and extension. Cold Reg. Sci. Technol., 8(3), $269-286$.

Li Jun, T. H. Jacka and W. F. Budd. 1996. Deformation rates in combined compression and shear for ice which is initially isotropic and after the development of strong anisotropy. Ann. Glaciol., 23, 247-252.

\section{SIR,}

Technique for improving core quality in intermediate-depth ice drilling

One of the factors that determine the quality of palaeoenvironmental records obtained by the analysis of ice cores is the quality of the ice core itself. Broken or cracked cores are difficult to sample even for simple measurements and can be unusable for contamination-sensitive studies such as trapped-air and trace metals. Core quality in intermediatedepth dry-hole drilling is discussed by Gillet and others (1984), Schwander and Rufli (1994) and Shoji (1994).

In the course of dry-hole mechanical drilling a $270 \mathrm{~m}$ 